\title{
The Plenoptic Camera as Wavefront Sensor for the VTT Solar Telescope
}

\author{
Noelia Martínez ${ }^{\mathrm{a}}$, Luis Fernando Rodríguez Ramos ${ }^{\mathrm{a}}$, Luz María Montoya ${ }^{\mathrm{a}}$, Iciar Montilla ${ }^{\mathrm{a}}$, and \\ Manuel Collados ${ }^{\mathrm{a}}$ \\ ${ }^{a}$ Instituto de Astrofísica de Canarias (IAC),Vía Láctea s/n, San Cristobal de La Laguna, Spain
}

\begin{abstract}
The plenoptic camera has the capability of generating images from as many different viewpoints as microlenses building its array, therefore it is possible to extract several images of the telescope aperture as viewed from those different viewpoints, and after processing them, estimate the incoming atmospheric wavefront. A customized plenoptic camera has been installed at the focus of the Vacuum Tower Telescope at the Teide Observatory in order to assess the feasibility of the plenoptic device as wavefront sensor for solar telescopes. While conventional sensors struggle when measuring phase in wide field-of-view and further from the optical axis, the plenoptic camera gets advantage of imaging extended objects as it could be the solar surface. Telescope results derived from sensing the atmospheric turbulence in a solar scenario by integrating the plenoptic camera in a real science environment are presented and the viability of this type of device as wavefront sensor for solar adaptive optics is hereby demonstrated.
\end{abstract}

Keywords: Solar Adaptive Optics, Plenoptic Wavefront Sensor, VTT

\section{INTRODUCTION}

Adaptive Optics (AO) systems measure and correct the aberrations caused by the atmosphere and have been extensively studied in both nocturnal and diurnal astronomical ground observations. While first night-time AO systems were built in the 1960's by military and aerospace communities, solar AO systems did not start to be developed until mid 1980's when Hardy performed the first Adaptive Optics Solar experiment at the Vacuum Tower Telescope at Sacramento Peak with a shearing interferometer as wavefront sensor. ${ }^{1}$ Technological development applied to solar AO has followed this very beginning by building and installing $\mathrm{AO}$ systems into several telescopes like the Vacuum Tower Telescope (VTT) or Gregor Telescope, both located at the Teide Observatory in Spain.

Diurnal Adaptive Optics deals with different challenges in comparison to nocturnal AO: worst day-time seeing, observations at visible wavelengths and the low-contrast extended objects the wavefront sensor shall work with (like solar granulation). ${ }^{2}$ Therefore, researching about wavefront sensors novel concepts could be fundamental in this field of expertise. Moreover, the Sun is an ideal object for the development of MCAO since solar structure provides multiple "guide stars" in any desired configuration.

Present paper will demonstrate the suitability of the plenoptic wavefront sensor for solar adaptive optics as well as presenting future implementations of a tomographic plenoptic wavefront sensor for solar telescopes. It is organised into the following sections: introduction to the plenoptic camera and the procedure to process plenoptic images, the experimental set-up which has been used to integrate the plenoptic WFS within the VTT telescope, and finally the achieved results and conclusions.

Further author information: (Send correspondence to N.M.)

N.M.: E-mail: noelia@iac.es

L.R.R.: E-mail: lrr@iac.es 


\subsection{The Plenoptic Camera}

The plenoptic camera makes profit of spatial multiplexing the light in order to achieve both spatial and angular information spreading over a single sensor: a microlenses array placed between the main lens and the sensor will fulfil this purpose, turned by Adelson and Wang ${ }^{3}$ into what it is known as the plenoptic camera, deeply developed afterwards by $\mathrm{Ng}^{4}$ Figure 1 shows the optical schematics of the plenoptic camera.

The functionality of interest in the plenoptic technology is its capacity of generating aperture images (in an optics system, i.e. a telescope) from different viewpoints, fact that allows the direct estimation of the wavefront gradients in this area.

The use of a plenoptic camera as a wavefront sensor was first described by Clare and Lane $(2005)^{5}$ for the case of point sources and recently it has been deeply analysed by Rodríguez-Ramos et al. (2008), ${ }^{6}$ Rodríguez-Ramos et al. (2009b), ${ }^{7}$ Rodríguez-Ramos et al. (2010), ${ }^{8}$ Montilla et al. (2010), ${ }^{9}$ Rodríguez-Ramos et al. (2012), ${ }^{10}$ and Rodríguez-Ramos $(2015)^{11}$ who have also studied the possibility of computing the tomography of the atmospheric turbulence and the height variations of Laser Guide Stars (LGSs) beacons.

Rodríguez-Ramos (2015), ${ }^{11}$ has proved the capabilities of the plenoptic camera as wavefront sensor as it has been demonstrated that it is able of measuring the gradients of the wavefront in the system aperture.

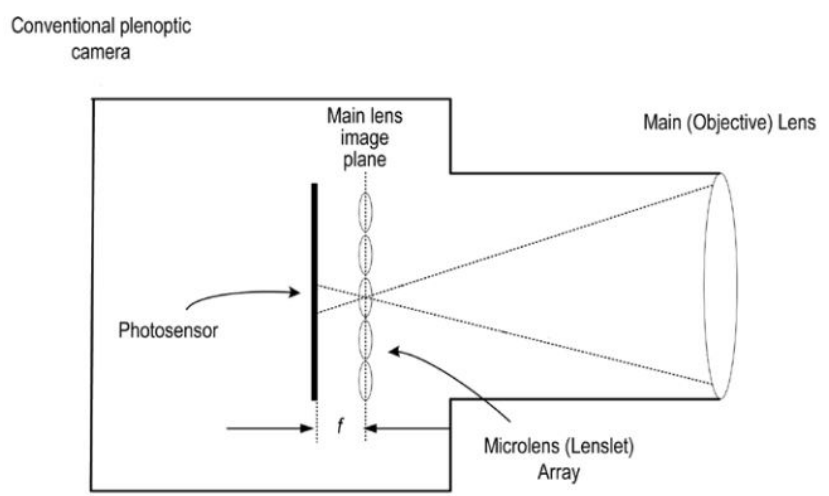

Figure 1: Optical schematics of the plenoptic camera. Notice the microlenses array located at the focal distance of the main lens, whose role is played by the telescope in the astronomical application. ${ }^{12}$

\subsection{Plenoptic Images Processing}

Every plenoptic image shall be deeply analysed in order to extract turbulence information. Procedure is divided into four phases: camera calibration, subaperture image generation, slopes computation and finally, wavefront reconstruction (Figure 2).

\subsubsection{Camera Calibration}

Some features of the plenoptic camera shall be well-known in order to reconstruct the wavefront from the acquired images, therefore a procedure for camera calibration is needed before starting any other image treatment.

Plenoptic calibration procedure consists of getting information regarding the positions of the multiple pupil images (from the lenslet array) and their relationship with the detector pixels. This knowledge is extracted from some physical parameters as they are the positions of the microlenses centers and consequently, the pitch as the inverse of the space in between one microlens and its neighbour, as well as the tilt between the pixel lines in the sensor and the microlenses within the array.

Pitch is estimated by a two-dimensional Fourier transform of the base image, whose module will present a peak in the pitch inverse spatial frequency and which will be detected by thresholding this spatial frequency into some lower and upper bounds. Simultaneously, the inclination is also calculated by this method and as a result of the 


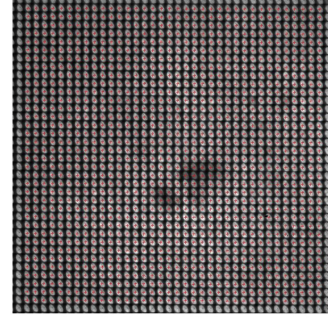

(a) Camera Calibration

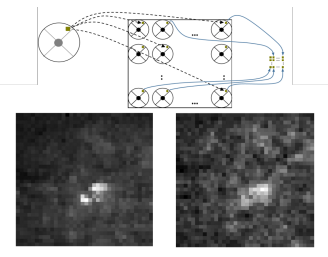

(b) Subaperture Image Generation

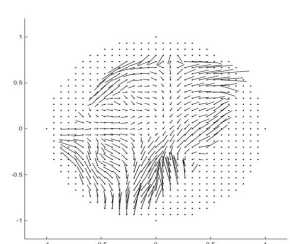

(c) Slopes Computation

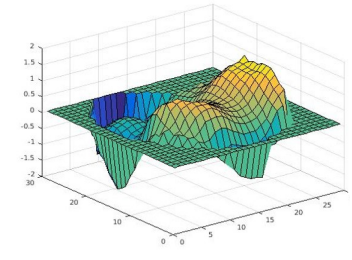

(d) Wavefront Reconstruction

Figure 2: Wavefront reconstruction process using a plenoptic camera.

pitch and inclination data, a grid with one microlens per square is built. Microlenses center positions are, then, obtained by computing the center of mass of the light inside each grid square.

Calibration procedure requires a bias corrected flat-field image to achieve optimum performance.

\subsubsection{Subaperture Image Generation}

The plenoptic camera allows the image synthesis of each aperture point independently and therefore, it is possible to make profit of all available techniques for the Shack-Hartmann sensor as the situation is similar as placing an aperture conjugated microlenses array. Figure 3 shows the image synthesis process by reordering the pixels related to the same position inside each microlens.

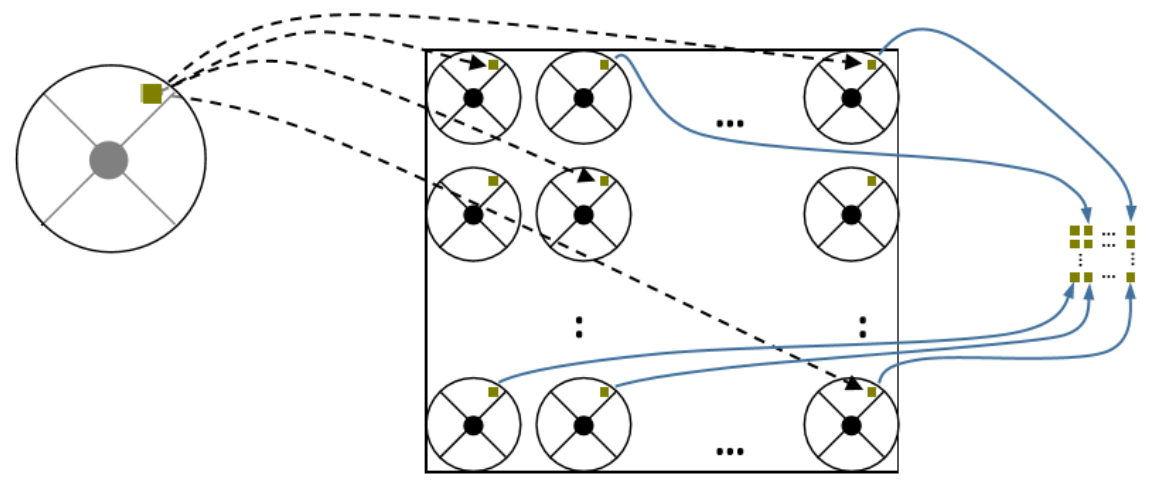

Figure 3: Subaperture image reconstruction. Left, aperture image of the telescope pupil; centre, plenoptic image on the sensor; right, procedure to synthesise the subaperture image corresponding to green viewpoint ${ }^{11}$

\subsubsection{Slopes Computation}

Once the subaperture images are extracted, the lateral displacements among them will be used to calculate local slopes by applying correlation techniques with a reference image (i.e. subaperture image corresponding to the central position of each microlens). For solar adaptive optics, the image behind each lenslet is extended and therefore, the displacement must be computed through a cross-correlation algorithm, instead of the conventional centroiding.

This information will grant, after a global integration, the wavefront extraction.

\subsubsection{Wavefront Reconstruction}

The wavefront reconstruction from the gradients will be performed by following the procedure in, ${ }^{11}$ which consists of the mathematical development in the Fourier domain of the wavefront:

$$
\phi\left(u_{1}, u_{2}\right)=F F T^{-1}\left(\alpha_{p q}\right)
$$


where $p$ and $q$ are the vertical and horizontal spatial frequencies, $\phi\left(u_{1}, u_{2}\right)$ is the aperture phase and $\alpha_{p q}$, the coefficients which minimise the mean square error are:

$$
\alpha_{p q}=\frac{i * p * F F T\left[S^{x}\left(u_{1}, u_{2}\right)\right]+i * q * F F T\left[S^{x}\left(u_{1}, u_{2}\right)\right]}{p^{2}+q^{2}}
$$

\section{EXPERIMENTAL SETUP}

During November 2015, several tests with a plenoptic camera were performed at the VTT solar telescope (see Table 1), located at the Teide Observatory (Canary Islands, Spain) with the aim of measuring atmospheric aberrations with solar images as reference.

The plenoptic WFS consisted of a Pulnix TM6200 camera plus a microlenses array at the lenslet focal length $(25 \mathrm{~mm})$ from the camera detector (Table 2), which was installed at the telescope focus. Additionally, a 589.5nanometer filter (Sodium wavelength) was used to avoid sensor over-exposure. Schematics of the experimental setup is represented in Figure 4. Due to the Sun elevation at this time of the year, VTT pupil was not fully illuminated (Figure 5).

Table 1: Vacuum Tower Telescope (VTT) optical parameters

\begin{tabular}{|l|l|}
\hline Telescope Diameter & $70 \mathrm{~cm}$ \\
\hline Focal Length & $46 \mathrm{~m}$ \\
\hline AO system & $36 \times 36 \mathrm{SH}$ at $70 \mathrm{~Hz}$ \\
\hline
\end{tabular}

Table 2: Plenoptic Camera optical parameters

\begin{tabular}{|l|l|}
\hline Camera Model & Pulnix TM6200 \\
\hline Sensor Size & 2048x2048 pixel \\
\hline Microlenses Array & $400 \mu m$ pitch and 25mm focal length \\
\hline
\end{tabular}

Several acquisition runs were performed in order to characterise the plenoptic camera as a wavefront sensor for solar AO: bias and flat field images were first captured to ease the device calibration procedure; sun spots were imaged with and without the adaptive optics system present in the telescope, and additionally, solar images with some optical aberrations added on purpose were also obtained with the aim of outlining the capabilities of the plenoptic camera to detect them.

\section{RESULTS AND DISCUSSION}

Results of November 2015 testing campaign at the VTT solar telescope are presented.

Artificial aberrations were introduced on purpose into the optical path by changing the shape of the deformable mirror (present in the current VTT AO system - see Table 1 for more AO details). The added aberrations have been investigated by imaging sunspots through the telescope (Figure 6 shows an example of a sunspot acquired by the plenoptic sensor). Following optical aberrations have been introduced into the system and deeply analysed: defocus, trefoil and spherical aberration.

First, defocus was introduced by moving forward and backward the telescope focus; Figure 7 shows the reconstruction of several degrees of defocus aberration after being sensed by the plenoptic camera. Right after, 


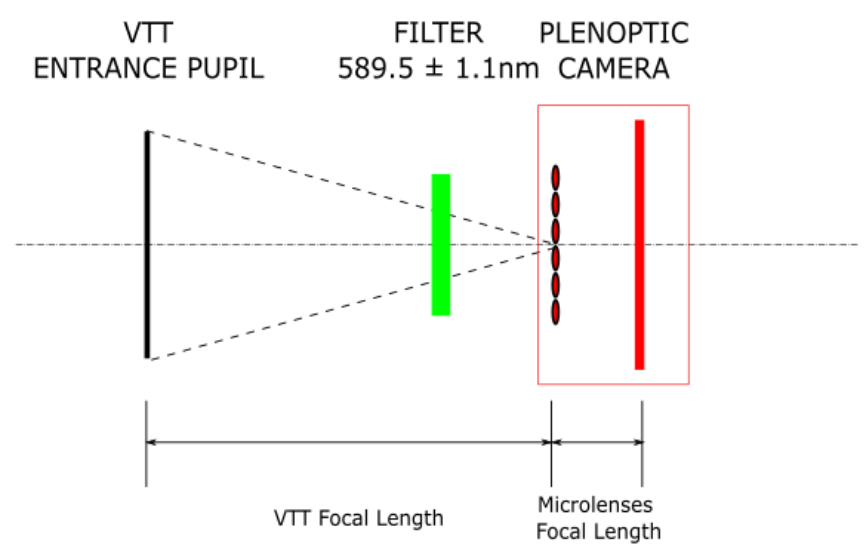

Figure 4: Schematics of the experimental setup installed at the VTT Solar Telescope during November 2015 test runs.

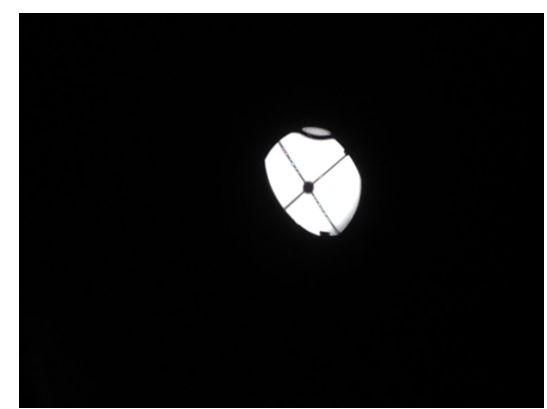

Figure 5: VTT partially illuminated pupil during November 2015 test runs due to the sun elevation at that time of the year.

trefoil aberration was created by applying the corresponding shape to the DM; reconstruction of trefoil is shown in Figure 8. Due to the partially illuminated pupil, only the trefoil peaks can be seen on the left side.

Spherical aberration could not be evaluated due to technical problems in the AO system which were discovered afterwards during the image processing phase.

In all wavefront reconstruction cases, in order to see only the artificial aberration of interest, 100 images have been averaged to suppress the atmospheric effect.

Besides the partially illuminated pupil, algorithms for plenoptic images processing have successfully reconstructed manually introduced aberrations, demonstrating the capability of this device as wavefront sensor for solar Adaptive Optics systems. Future tasks will assess the functionality of the plenoptic camera as tomographic wavefront sensor to be considered a possible candidate for the MCAO systems in solar telescopes. 


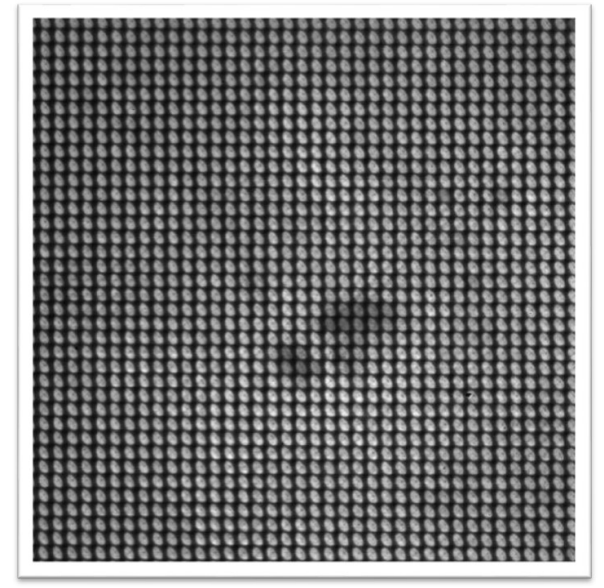

Figure 6: Plenoptic image of a sunspot acquired through the VTT solar telescope.

\section{ACKNOWLEDGMENTS}

The authors would like to thank the Instituto de Astrofísica de Canarias (IAC) for funding this research.

\section{REFERENCES}

[1] Hardy, J., "Solar isoplanatic patch measurements," in [Solar instrumentation: What's next?], Dunn, R. B., ed., 421 (mar 1981).

[2] Rimmele, T. R., "Solar adaptive optics," (2000).

[3] Adelson, E. H. and Wang, J. Y. A., "Single lens stereo with a plenoptic camera," IEEE Transactions on Pattern Analysis and Machine Intelligence 14, 9 (1992).

[4] Ng, R., Digital Light Field Photography, PhD thesis, Stanford University (2006).

[5] Clare, R. and Lane, R., "Wave-front sensing from subdivision of the focal plane with a lenslet array," $J$. Opt. Soc. Am. A 22, 117-125 (2005).

[6] Rodríguez-Ramos, J., Lüke, J., López, R., Marichal-Hernández, J., Montilla, I., Trujillo-Sevilla, J., Femenía, B., Puga, M., López, M., Fernández-Valdivia, J., Rosa, F., Dominguez-Conde, C., Sanluis, J., and L.F, R.R., "Wavefront and distance measurements using the cafadis camera in astronomical telescopes," Proc. SPIE Adaptive Optics Systems (2008).

[7] Rodríguez-Ramos, L., Martín, Y., Díaz, J., Piqueras, J., and Rodríguez-Ramos, J., "The plenoptic camera as a wavefront sensor for the european solar telescope (est)," Proc. SPIE 7439, Astronomical and Space Optical Systems $\mathbf{7 4 3 9 0 I}$ (2009).

[8] Rodríguez-Ramos, J., Femenía, B., Montilla, I., Rodríguez-Ramos, L., Marichal-Hernández, J. G., Lüke, J., López, R., Díaz, J., and Martín, Y., "The cafadis camera: a new tomographic wavefront sensor for adaptive optics," in [1st AO4ELT conference], 05011 (2010).

[9] Montilla, I., Reyes, M., Femenía, B., and Rodríguez-Ramos, J., "Multiconjugate adaptive optics with plenoptic cameras and the fourier transform reconstructor," Proc. SPIE 7736, Adaptive Optics Systems II $\mathbf{7 7 3 6 4 1}$ (2010).

[10] Rodríguez-Ramos, L., Montilla, I., Fernández-Valdivia, J., Trujillo-Sevilla, J., and Rodríguez-Ramos, J., "Concepts, laboratory, and telescope test results of the plenoptic camera as a wavefront sensor," Proc. SPIE 8447, Adaptive Optics Systems III 844745 (2012).

[11] Rodríguez-Ramos, L., [Utilización de la cámara plenóptica como sensor de frente de onda para Óptica Adaptiva en Astrofísica], Phd Thesis. Universidad de La Laguna (2015).

[12] Georgiev, T. and Lumsdaine, A., "Focused plenoptic camera and rendering," Journal of Electronic Imaging 19(2), 021106-021106-11 (2010). 


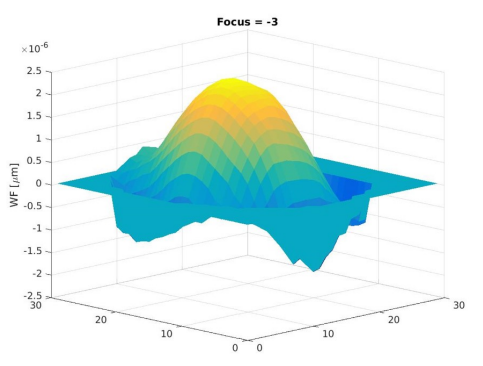

(a)

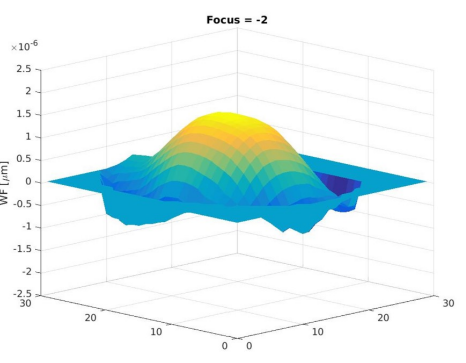

(b)

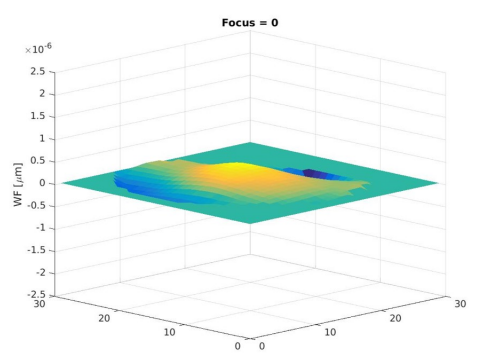

(d)

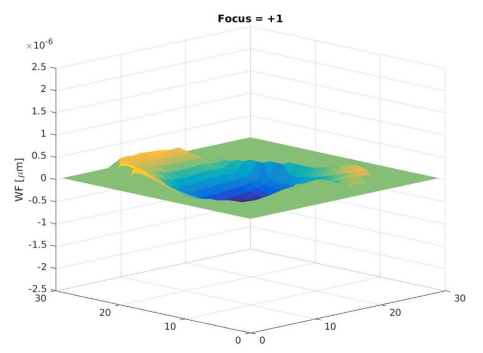

(e)

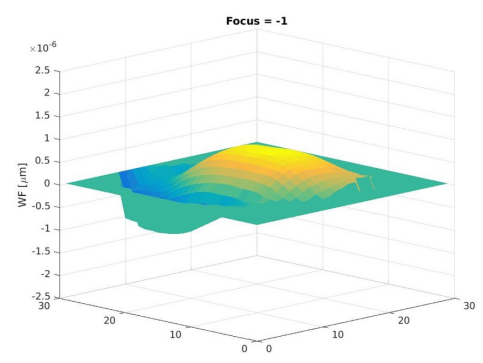

(c)

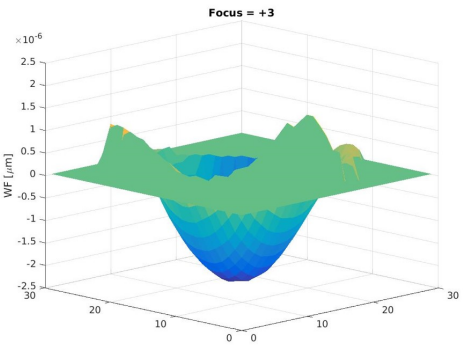

$(\mathrm{g})$

Figure 7: Defocus aberration created by changing the telescope focus and reconstructed from the plenoptic images. 


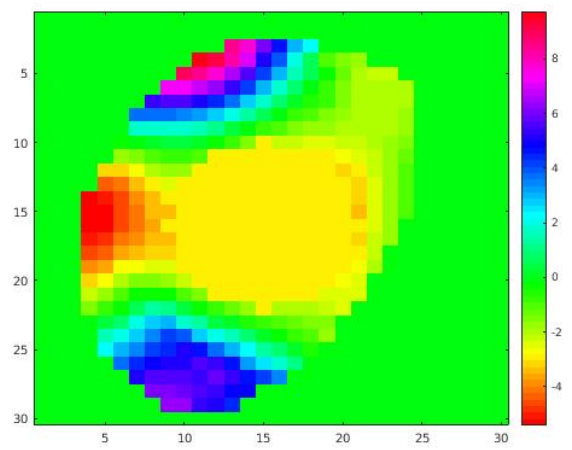

(a) Trefoil aberration created by changing the shape of the DM in the VTT AO system and reconstructed from the plenoptic images

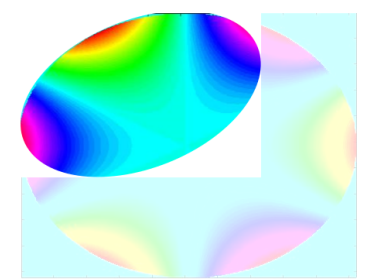

(b) Trefoil Zernike Representation and the area corresponding to the partial pupil

Figure 8: Trefoil aberration 